BULLETIN OF THE

AMERICAN MATHEMATICAL SOCIETY

Volume 80, Number 4, July 1974

\title{
SETS OF COLORINGS OF CIRCUITS
}

BY JAMES W. SCHLESINGER

Communicated by Gian-Carlo Rota, November 6, 1973

1. Introduction. A circuit $\Gamma$ is a triangulation of the one-dimensional sphere $S^{1}$. It shall have as its set of vertices $\Gamma_{0}=Z_{k}=\{0,1, \cdots, k-1\}$, and as its set of one-simplices $\Gamma_{1}=\left\{\sigma_{j}=(j-1, j) \mid j=1,2, \cdots, k\right\}$. A coloring of $\Gamma$ is a zero-dimensional cochain $c^{0} \in C^{0}\left(\Gamma, Z_{2} \oplus Z_{2}\right)$ whose coboundary is "nowhere zero", i.e. $\delta c^{0}\left(\sigma_{j}\right) \neq 0$ for all $\sigma_{j} \in \Gamma_{1}$. A set $K$ of colorings of $\Gamma$ is realizable as a set of admissible colorings if there is a triangulated two-dimensional disk $D$ with boundary $\Gamma$ such that the restriction homomorphism

$$
j^{\#}: C^{0}\left(D, Z_{2} \oplus Z_{2}\right) \rightarrow C^{0}\left(\Gamma, Z_{2} \oplus Z_{2}\right)
$$

(induced by the inclusion $j: \Gamma \rightarrow D$ ) takes the colorings of $D$ onto $K$.

Let $\psi(k)$ be the minimum cardinality of a set $K$ which is realizable as a set of admissible colorings.

REMARK 1. $\psi(k)=0$ if and only if the four color conjecture is false.

The conjecture of Albertson and Wilf $[\mathbf{1}] . \quad \psi(k)=3 \cdot 2^{k}$ for $k=3,4, \cdots$.

Comment 1. Since $3 \cdot 2^{k}$ is the number of colorings of any disk $D$ with no interior vertices and $k$ vertices in $\Gamma_{0}=D_{0}$, we conclude $3 \cdot 2^{k} \geqq \psi(k)$.

Comment 2. It is not known whether the four color conjecture implies the Albertson-Wilf conjecture for $k>6$. (It does for $k=3,4,5$ and 6 [1].)

In [1], Albertson and Wilf announce:

THEOREM 1. If the four color conjecture holds then

$$
\psi(k) \geqq(4 !) F_{k-1} \geqq C((1+\sqrt{ } 5) / 2)^{k}
$$

where $F_{k}$ is the kth Fibonacci number.

By generalizing the notion of a set of admissible colorings of $\Gamma$ to the notion of a complete set of colorings of $\Gamma$, one can prove by induction on $k$ :

THEOREM 2. If the four color conjecture holds then

$$
\begin{aligned}
& \psi(k)>4 \cdot 3^{k / 2} \quad \text { if } k \text { is even, } \\
& >8 \cdot 3^{(k-1) / 2} \text { if } k \text { is odd. }
\end{aligned}
$$

AMS (MOS) subject classifications (1970). Primary $05 C 15$.

Key words and phrases. Four color problem, set of all colorings. 
2. Coboundaries of colorings. Let $D$ be a triangulated two-dimensional disk with boundary $\Gamma$. Since $D$ is connected, $H^{0}\left(D, Z_{2} \oplus Z_{2}\right) \approx Z_{2} \oplus Z_{2}$. Hence there are exactly four colorings corresponding to each nowhere zero one-dimensional cobounding cocycle. All of the sets of colorings that we consider will contain all four colorings with a given coboundary if the set contains any one of them. Thus we can consider the sets of coboundaries of colorings as easily as the sets of colorings.

The disk $D$ is contractible so $H^{1}\left(D, Z_{2} \oplus Z_{2}\right) \approx 0$. Hence the group of cocycles $Z^{1}\left(D, Z_{2} \oplus Z_{2}\right)$ is equal to the group of cobounding cocycles $B^{1}\left(D, Z_{2} \oplus Z_{2}\right)$. [ $H^{1}\left(\Gamma, Z_{2} \oplus Z_{2}\right) \approx Z_{2} \oplus Z_{2}$. In this case the cobounding cocycles are characterized by the sum of all values being zero.]

Notation. $Z_{2} \oplus Z_{2}=\left\{0, e_{1}, e_{2}, e_{3}\right\}$ with the obvious addition.

If $z$ is a nowhere zero cocycle on $D$ and $\tau^{2}$ is a two-simplex with faces $\alpha, \beta$ and $\gamma$, then $z(\alpha)+z(\beta)+z(\gamma)=0$. Hence $z$ assigns the three values $e_{1}, e_{2}$ and $e_{3}$ to the faces $\alpha, \beta$ and $\gamma$ of $\tau^{2}$. Let us suppose that $z(\beta)=e_{3}$. We may hold the value $e_{3}$ fixed and interchange the values $e_{1}$ and $e_{2}$ on $\alpha$ and $\gamma$. This change will propagate along a $Z_{2}$ cocycle which contains either no one-simplexes from $\Gamma_{1}$ or exactly two one-simplexes from $\Gamma_{1}$.

REMARK 2. Let $z$ be a nowhere zero cocycle on $D$ and $e_{i}$ a fixed value in $Z_{2} \oplus Z_{2}$. For each $\sigma_{j}$ in $\Gamma_{1}$ with $z\left(\sigma_{j}\right) \neq e_{i}$, there is a uniquely determined $\sigma_{j^{\prime}} \in \Gamma_{1}$ and $z^{\prime}$ a nowhere zero cocycle on $D$ such that:

(i) $j^{\prime} \neq j$.

(ii) For every $\alpha \in D_{1}, z^{\prime}(\alpha)=e_{i}$ if and only if $z(\alpha)=e_{i}$.

(iii) For every $\sigma_{l} \in \Gamma_{1}$

$$
\begin{aligned}
z^{\prime}\left(\sigma_{l}\right) & =z\left(\sigma_{l}\right)+e_{i} & & \text { if } l=j, j^{\prime} \\
& =z\left(\sigma_{l}\right) & & \text { otherwise. }
\end{aligned}
$$

The pairing $\sigma_{j} \leftrightarrow \sigma_{j^{\prime}}$ is called a planar change diagram for $j^{\#}(z)$ and $e_{i}$. $\left[j^{\#}(z) \in Z^{1}\left(\Gamma, Z_{2} \oplus Z_{2}\right)\right.$.] It satisfies:

(i) If $\sigma_{j} \leftrightarrow \sigma_{j^{\prime}}$, then neither $z\left(\sigma_{j}\right)=e_{i}$ nor $z\left(\sigma_{j^{\prime}}\right)=e_{i}$. Furthermore if $z\left(\sigma_{j}\right) \neq e_{i}$ then $\sigma_{j}$ belongs to a pair.

(ii) If $\sigma_{j^{\prime}} \leftrightarrow \sigma_{j^{\prime}}$ and $\sigma_{l^{\prime}} \leftrightarrow \sigma_{l^{\prime}}$ then $\sigma_{l}$ and $\sigma_{l^{\prime}}$ lie on the same arc between $\sigma_{j}$ and $\sigma_{j^{\prime}}$.

Let $z$ be a nowhere zero cobounding cocycle on $\Gamma$ and let $P$ be a planar change diagram for $z$ and $e_{i}$. With each set of pairs of $P$ we can associate a nowhere zero cobounding cocycle $z^{\prime}$ on $\Gamma$. This association is called the action of $P$ on $z$. If $z$ is in some set $L$ of cocycles and $z^{\prime} \in L$ for all sets of pairs of $P$ then we say $L$ is closed under the action of $P$.

Definition. A complete set $K$ of colorings of $\Gamma$ corresponds to a set $\delta K$ of nowhere zero cobounding cocycles with the properties:

(i) $K$ is invariant under the action of the six automorphisms $\nu: Z_{2} \oplus$ $Z_{2} \rightarrow Z_{2} \oplus Z_{2}$. 
(ii) For each $z \in \delta K$ and $e_{i}$ there is a planar change diagram $P$ so that $\delta K$ is closed under the action of $P$.

3. Induced sets. A nondegenerate simplicial map $f: E \rightarrow F$ induces a homomorphism: $f^{\#}: B^{1}\left(F, Z_{2} \oplus Z_{2}\right) \rightarrow B^{1}\left(E, Z_{2} \oplus Z_{2}\right)$, which preserves the property of being nowhere zero. In general, however, complete sets of colorings on circuits are not preserved. [Let $f: \Gamma^{\prime} \rightarrow \Gamma$ be a two-fold covering.]

4. Potted trees. A potted tree is a contractible simplicial complex with no more than one two-dimensional simplex. A circuit $\Gamma$ is properly mapped to a potted tree $T$ if $f: \Gamma \rightarrow T$ is a nondegenerate simplicial map such that for each $\alpha \in T_{1}, f^{-1}(\alpha)$ has exactly one or exactly two elements depending upon whether $\alpha$ is the face of a two simplex or not.

REMARK 3. If $\Gamma$ is properly mapped to the potted tree $T$ then $T_{1}$ has $k / 2$ or $(k+3) / 2$ elements. The set $L$ of all colorings of $T$ has $n(k)$ elements and $f^{\#}(L)$ is a complete set of colorings of $\Gamma$ of cardinality $n(k)$ where

$$
\begin{aligned}
n(k) & =4 \cdot 3^{k / 2} & & \text { if } k \text { is even, } \\
& =8 \cdot 3^{(k-1) / 2} & & \text { if } k \text { is odd. }
\end{aligned}
$$

Comment 3. $n(k) \leqq 2 \cdot n(k-1)$ [equality when $k$ is odd]; $n(k)=$ $3 \cdot n(k-2)$.

A set $K$ of colorings of $\Gamma$ is realizable as induced by a potted tree if there exists a proper map $f: \Gamma \rightarrow T$ such that $K=f^{\#}(L)$.

5. Outline of the proof of Theorem 2. From a circuit $\Gamma$, we can form a circuit $\Gamma^{\prime}$ by deleting the open star of the vertex 1 and by inserting a one-simplex $(0,2)$. We can also form a circuit $\Gamma^{\prime \prime}$ by performing the same deletion and identifying the vertices 0 and 2 . Since every nowhere zero cobounding cocycle $z$ on $\Gamma$ induces either a cocycle $z^{\prime}$ on $\Gamma^{\prime}$ or a cocycle $z^{\prime \prime}$ on $\Gamma^{\prime \prime}$, a complete set of colorings $K$ on $\Gamma$ induces complete sets $K^{\prime}$ and $K^{\prime \prime}$ on $\Gamma^{\prime}$ and $\Gamma^{\prime \prime}$, respectively.

We prove by induction on the number $k$ of vertices of $\Gamma$, that $K$ has fewer than $n(k)$ elements only if $K$ is empty. This follows from two inequalities. First the number of elements in $K^{\prime \prime}$ is less than or equal to one third the number of elements in $K$. Secondly, if $K^{\prime \prime}$ is empty then the number of elements in $K^{\prime}$ is less than or equal to half the number of elements in $K$. In essentially the same way we can prove that if $K$ has exactly $n(k)$ elements then $K$ can be realized as induced by a potted tree.

\section{REFERENCE}

1. M. O. Albertson and H. S. Wilf, Boundary values in chromatic graph theory, Bull. Amer. Math. Soc. 79 (1973), 464.

215 Clark Road, Brookline, Massachusetts 02146 